\title{
ARTICLE
}

\section{Estimation of photon yield in liquid scintillation counter by using Geant4 Monte Carlo simulation}

\author{
Tsukasa Aso ${ }^{a^{*}}$, Masanori Hara ${ }^{\mathrm{b}}$, Miki Shojic ${ }^{\mathrm{c}}$, Takayoshi Furusawa ${ }^{\mathrm{d}}$, Tomoyuki Yoshimura ${ }^{\mathrm{d}}$ and Yuka Kato $^{\mathrm{d}}$ \\ ${ }^{a}$ National Institute of Technology, Toyama College, 1-2 Neriya Ebie, Imizu, Toyama, 933-0293, Japan; ${ }^{b}$ Hydrogen Isotope Center, \\ Univeristy of Toyama, 3190 Gofuku, Toyama, 930-8555, Japan; ' Life Science Research Center, University of Toyama, 2630 \\ Sugitani, Toyama, 930-0194, Japan; ${ }^{d}$ Hitachi, Ltd., 6-22-1 Mure, Mitaka-shi, Tokyo, 181-8622, Japan
}

Liquid scintillation counter (LSC) is an efficient technique for quantitative measurement of radioactivity in low energy beta decay nuclide. However, the detection efficiency varies with quench effect that caused by missing energy in the conversion from the energy of beta-ray to the intensity of scintillating photons. For the purpose of investigating the effect of photon yield on efficiency and quench level, the Geant 4 simulation has been performed. The simulation modeled LSC-5100 manufactured by Hitachi Ltd. It consists of two photomultipliers (PMTs) placed around a sample vial in an optical chamber, and the activity is determined by using the count of double coincidence event in two PMTs. The simulation accounts for radioactive decay, electromagnetic interactions, scintillation and optical photon processes. The LSC spectrum was calculated from optical photons reached at the PMT windows. The photon yields in the simulation were estimated to reproduce the measured spectra in $\mathrm{C}-14$ and $\mathrm{H}-3$ standard quenched samples of Ultima Gold provided by PerkinElmer Co. Ltd. The calculated efficiencies in the photon yields well reproduced the measured efficiencies. In addition, the estimated photon yields were verified in the simulation of the external standard channel ratio (ESCR) with Cs-137 source. The ESCRs in the simulation reproduced the measured ESCRs as well.

Keywords: Geant4 Monte Carlo simulation; liquid scintillation counter; counting efficiency; H-3; C-14; external standard channel ratio; scintillation photon yield

\section{Introduction}

Liquid scintillation counter (LSC) has been used as a very efficient technique for a quantitative measurement of radioactivity. A sample is dissolved in a liquid scintillation cocktail so that it acts as a $4 \pi$ solid angle detector. This characteristic is suitable for efficiently detecting low energy beta rays from radionuclides such as tritium (H-3). The activity of sample is quantified by counting number of flashes of scintillation lights in LSC system. However, the count rate varies with the detection efficiency that is caused by energy loss in the conversion from the energy of beta ray to the intensity of scintillation photons. The phenomenon is called as a quench. Therefore, the compensation of the loss in count rate is essential to determine the sample's activity.

There are many techniques to determine the detection efficiency [1]. One of methods is to measure a set of standard samples, in which each contains the scintillation cocktail and a well-known amount of activity of the nuclide under study but different amount of a quenching agent. Consequently, the efficiency curve is obtained as a function of quenching indicator. The external standard channel ratio (ESCR) is measured as a quenching indicator for each sample with an external standard source included in the LSC system. However, such standard samples are commercially available only for $\mathrm{C}-14$ and $\mathrm{H}-3$. The preparation of the standard sample for the other radionuclide is not easy, because it requires meticulous source preparation techniques, and also it is very difficult for short lived radionuclides.

For the purpose of investigating the detection efficiency on quench effect in LSC, a Geant4 [2-4] based Monte Carlo simulation has been applied. Since the loss of detection efficiency is caused by the degradation of photon yield due to quench, we estimated the photon yield in each ESCR for C-14 and H-3. The photon yield was determined by comparing the LSC spectra between the simulation and the measurement. The obtained photon yields were also applied to simulate a Compton electron spectrum to calculate ESCR. The calculated ESCRs were verified with the measurements.

*Corresponding author. Email: aso@nc-toyama.ac.jp 


\section{Materials and methods}

\subsection{Measurement}

The measurement of LSC spectra has been performed with LSC-5100 manufactured by Hitachi Ltd., which is a LSC system for activity measurements based on liquid scintillation counting. It consists of two photomultipliers (PMTs) placed around a sample vial in an optical chamber. The activity is determined by using the count of double coincidence event in two PMTs.

The LSC spectra of C-14 and H-3 were obtained by measuring a set of standard samples of Ultima Gold provided by PerkinElmer Co. Ltd. Each detection efficiency was derived by taking the ratio of the count rate in the measurement to the calculated activity. The ESCR of each sample was automatically measured too. The LSC-5100 employs a Cs-137 point source to obtain a LSC spectrum of Compton electrons and calculates the ESCR. The maximum energy of the Compton electron is about $478 \mathrm{keV}$, which is larger than the maximum energy of beta ray in C-14. The detection efficiencies of the standard samples were ranging from $95.9 \%$ to $46.1 \%$ and from $53.3 \%$ to $1.2 \%$ for $\mathrm{C}-14$ and $\mathrm{H}-3$ samples, respectively. The ESCRs were ranging from 4.55 to 0.47 and from 4.23 to 0.40 for $\mathrm{C}-14$ and $\mathrm{H}-3$ samples, respectively.

\subsection{Simulation model}

The LSC simulation has been developed on the Geant4 Monte Carlo simulation toolkit, version 10.1 patch-01. The simulation modeled LSC-5100. The geometric parameters were taken from the datasheet. The simulation accounts for radioactive decay, electromagnetic interactions, scintillation process and the propagation of optical photon. The optical properties were assigned to materials and boundaries of the geometries. The schematic view of the geometry is shown in Figure 1.

A glass vial was filled with Ultima Gold liquid scintillation cocktail of PerkinElmer Co. Ltd. The cocktail contains 2,5-Diphenyloxazole (PPO) and 1,4-Bis (2-methylstyryl) benzene (bis-MSB) as solutes. The atomic composition was taken from [5]. The refractive indices of glass and scintillation cocktail were set to 1.49 and 1.53 , respectively. As a primary event, a radionuclide, $\mathrm{C}-14$ or $\mathrm{H}-3$, was randomly generated in the scintillation cocktail volume and a beta ray was produced by radioactive decay. Then the scintillation photons were generated isotropic directions along the track segment. The yield of scintillating photons in the energy loss during the step length for the C-14 sample of $\mathrm{ESCR}=4.55$ was assumed to 10 photons $/ \mathrm{keV}$. The spectral response of scintillation lights by electrons in Ultima Gold was taken from [6]. The refraction and reflection of optical photons at the boundary of materials were simulated with the refractive index of the interface, while the reflectivity at the inner surface of optical chamber was assigned according to the LSC-5100 datasheet to account for the reflector paint.
According to the quantum efficiency of PMT, only $25 \%$ of photons reached at the PMT windows were randomly sampled. Then, photons were converted and integrated to form a PMT signal by referring the wavelength dependent sensitivity table of PMT. The double coincidence was emulated by requiring at least one photon in both PMTs. The final LSC signal was calculated as a sum of both PMT signals. Since the total gain of PMTs and the readout circuit were not clear, we estimated a spectral matching factor so that the simulated spectrum coincides with the measured LSC spectrum of the C-14 sample in ESCR $=4.55$, in which the quenching effect can be assumed to be very small. The spectral matching factor was applied to all simulations as a fixed coefficient.

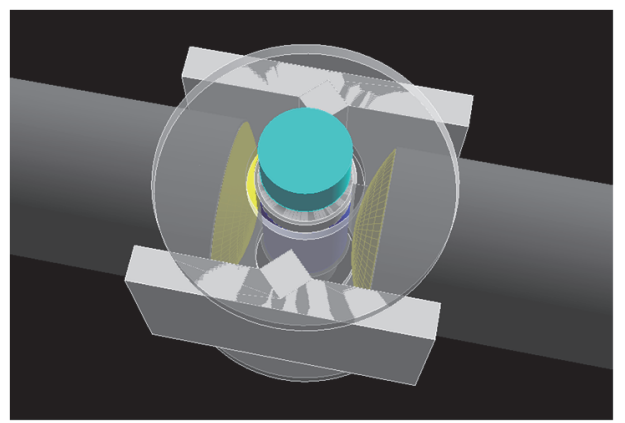

Figure 1. The schematic view of LSC-5100 geometry in the simulation.

\subsection{Quenching}

The quenching concerning to the standard samples is categorized into chemical quenching and ionization quenching. The former is caused by the presence of added substances acting as chemical scavengers of excited molecules of the solvent, while the latter is a result of high ionization density along the track of a charged particle in the scintillator. The fluorescent yield of the scintillator, $L(E)$, is given by

$$
\mathrm{L}(\mathrm{E})=\eta_{0} E Q(E)
$$

where $E, \eta_{0}$ and $Q(E)$ are the energy of incident beta-ray, the photon yield emitted per unit of energy, and the ionization quenching function, respectively. The $Q(E)$ is expressed with the Birks semi-empirical formula by

$$
Q(E)=\frac{1}{E} \int_{0}^{E} \frac{d E}{1+k B\left(\frac{d E}{d x}\right)} .
$$

Here $d E / d x$ is the stopping power of the incident beta-ray and $k B$ is the ionization quenching parameter known as Birks parameter. The photon yield $\eta_{0}$ includes the effect of chemical quenching. The Stern-Volmer quench model [7] describes the chemical quenching by accounting for quenching concentration. The emission probability is simply given by the ratio of fluorescence intensities with and without quenchers.

Because the purpose of this study is to investigate the effect of photon yield on the detection efficiency, we focused on the average photon yield of $\eta_{0}$ over the energy range of incident beta-rays by assuming $k B=0$. Therefore, the estimated photon yield $\eta_{0}$ includes both 
effects from chemical quenching and ionization quenching.

\subsection{Analysis}

\subsubsection{Liquid scintillation counter spectrum}

In order to estimate a photon yield and a detection efficiency, we compared the LSC spectra between the simulation and the measurement in each sample. The photon yield was adjusted in the simulation so that the spectra in the simulation and the measurement coincides each other. The detection efficiency was calculated by taking a ratio of the number of double coincidence events to the number of decays in the simulation.

\subsubsection{External standard channel ratio}

The ESCR is calculated from the channel number, $R_{\gamma}$, which divides the count ratio of the Compton electron spectrum into three to one. The ESCR is given by

$$
E S C R=K \times R_{\gamma}
$$

where $K$ is a constant and equals to $0.01 /$ channel in LSC-5100. In order to reduce the contribution of backgrounds such as chemiluminescence, the lowest level (L.L.) is applied to the LSC spectrum in the ESCR calculation. The L.L. is set to 30 channel in LSC-5100. Since one channel corresponds to $0.5 \mathrm{keV}, K$ and L.L correspond to $0.02 / \mathrm{keV}$ and $15 \mathrm{keV}$, respectively.

The spectrum was simulated by placing a Cs-137 point source just under the sample vial. The ionization quench effect was assumed to be similar for the energy range of electrons in $\mathrm{C}-14$ and $\mathrm{Cs}-137$, and the photon yields determined in the spectral analysis of C-14 was applied to the Compton electrons of Cs-137. Then, the $R_{\gamma}$ in the simulation $\left(R_{\gamma}^{\mathrm{Sim} .}\right)$ was calculated from the simulated Compton electron spectrum for each sample. The dependence of $R_{\gamma}^{\text {Sim. }}$ on the photon yield was fitted with a linear function. The relation between the ESCRs in the measurement (ESCR $\left.{ }^{\text {Meas. }}\right)$ and the corresponding photon yields determined in the simulation were also fitted with a linear function. Since the linear functions have in common the photon yield, the relation between $R_{\gamma}^{\text {Sim. }}$ and ESCR $R^{\text {Meas. }}$ is derived as

$$
E S C R^{\text {Meas. }}=a \times R_{\gamma}^{\text {Sim. }}+b
$$

where $a$ and $b$ are constant. However, these values are changed with the choice of L.L value. Therefore, the analysis was repeated by varying the L.L value to verify whether the obtained $a$ and $b$ are consistent with the parameters in LSC-5100, for instance it should be $a=0.02 / \mathrm{keV}$ and $b=0$, respectively.

\section{Results}

\subsection{Counting efficiency and photon yield}

Figure 2 shows example LSC spectra for C-14 and $\mathrm{H}-3$ after adjusting the photon yield in the simulation to coincide with the measured spectrum. As shown in the figure, the simulated spectrum well reproduces the measured spectrum in both $\mathrm{C}-14$ and $\mathrm{H}-3$.

Figure 3 shows the comparison of efficiencies between the simulations and the measurements. The estimated efficiencies are consistent with the measurements for both $\mathrm{C}-14$ and $\mathrm{H}-3$, respectively.
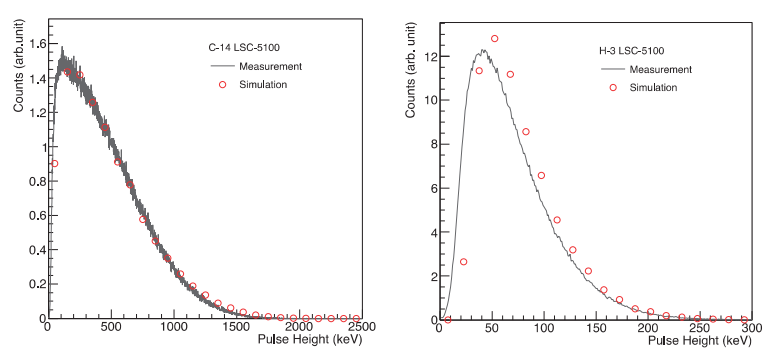

Figure 2. Example LSC spectra for a C-14 sample of ESCR $=3.18$ (Left) and a H-3 sample of ESCR=3.60 (Right). Here the photon yields were chosen to $7.2 / \mathrm{keV}$ and $6.2 / \mathrm{keV}$ for C-14, and H-3.

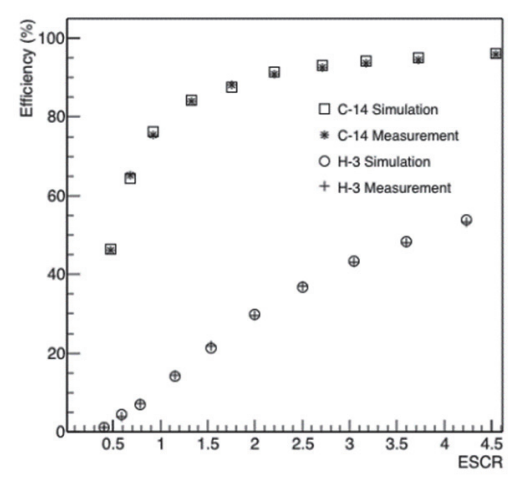

Figure 3. Dependence of detection efficiency on ESCR of measurement for $\mathrm{C}-14$ and $\mathrm{H}-3$, respectively.

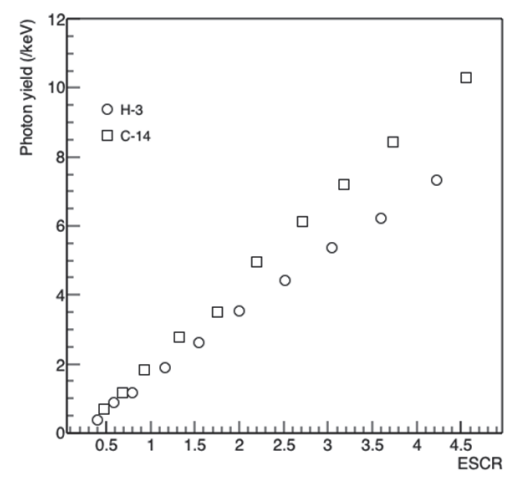

Figure 4. Relation between photon yield and ESCR of measurement for C-14 and H-3.

Figure 4 shows the relation between the photon yields and the ESCRs of measurement for C-14 and H-3. Even the same ESCR, the photon yields of $\mathrm{H}-3$ are smaller than that of C-14. This is presumably due to the effect of ionization quench that reduces the photon yield when the linear energy transfer is large.

\subsection{External standard channel ratio}

We repeated the ESCR calculation in the simulated Cs-137 spectra by varying the L.L value from $0 \mathrm{keV}$ to $23 \mathrm{keV}$. Figure 5 demonstrates the relations between ESCR ${ }^{\text {Meas. }}$ and $R_{\gamma}^{\text {Sim. }}$ at three different L.L values. 


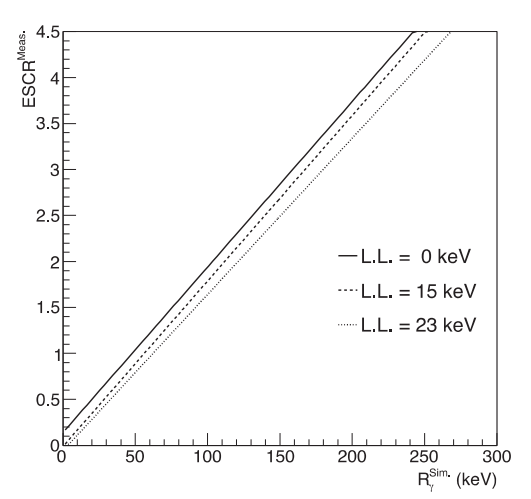

Figure 5. Relations between $E S C R^{\text {Meas. }}$ and $R_{\gamma}^{\text {Sim. in three }}$ different L.L. value.

When the L.L. was set to zero, the parameter $b$ became 0.14 . The parameter $b$ decreased by choosing larger value of L.L. and closed to zero at L.L $=15 \mathrm{keV}$. The parameter $a$ was rather stable in the choice of L.L. value. It was about $0.018 / \mathrm{keV}$. The value of $K$ in the simulation is about $10 \%$ smaller than the value in LSC-5100. This is probably because the simulation did not include a brass window of Cs-137 source capsule. Such additional materials will attenuate gamma-rays and eventually increase the low energy signals, and consequently, decrease $R_{\gamma}$. These results demonstrate that the calculation of ESCR in the simulation is applicable to the ESCR in LSC-5100.

\section{Discussion}

The main source of the uncertainty for the calculation of the photon yield and the detection efficiency is the systematic error in comparing the spectra between the simulation and measurement. We studied the influence by checking the pulse height differences at two featuring points [8]. The uncertainty in the photon yield was estimated to be less than $9 \%$, while the corresponding uncertainty in the detection efficiency was estimated to be less than $1.8 \%$.

P. Skarstad et al. [9] determined the scintillation efficiency in excitation of a benzene solution for C-14 beta-ray. By assuming the PPO solution, the average photon yield can be equated to $13 / \mathrm{keV}$, while we have assumed the photon yield of $\mathrm{C}-14$ unquenched sample to be $10 / \mathrm{keV}$. Because we introduced the spectral matching factor to calibrate the spectrum accounting for the gain of PMT and the readout circuit in LSC, the difference of photon yield had been compensated by this spectral matching factor. Additional measurements about the gain of PMT and the readout circuit are important to determine the absolute photon yield of the scintillator.

\section{Conclusion}

We have demonstrated the simulation of the liquid scintillation counter of LSC-5100. The photon yields of C-14 and H-3 in each ten different quenching samples were estimated. The detection efficiencies at the estimated photon yields were good agreement with the measured efficiencies. We have also verified the ESCR calculation in the simulation by applying the estimated photon yield of $\mathrm{C}-14$. These results show that the photon yield can be used as a common parameter in the simulation to estimate the detection efficiency and the LSC spectrum.

\section{Acknowledgements}

The authors wish to thank Yuki Tsubouchi and Weerasekara Helanka for the helpful contributions to the data analysis. This work was partly supported by JSPS KAKENHI Grant number 26610065 and 17K07006.

\section{References}

[1] M.F. L'Annuziata and M.J. Kessler, Liquid scintillation analysis: principles and practice. In: L'Annuziata MF (ed), Handbook of Radioactivity Analysis, Academic press, California.

[2] S. Agostinelli, J. Alison, K. Amako, et al., Geant4 -a simulation toolkit, Nucl. Instrum. and Meth. A506 (2003), pp. 250-303.

[3] J. Allison, L. Amako, J. Apostolakis, et al., Geant4 developments and applications, IEEE Trans. on Nucl. Sci. 53 (2006), pp. 270-278.

[4] J. Allison, K. Amako, J. Apostolakis, et al., Recent developments in Geant4, Nucl. Instrum. and Meth. A835 (2016), pp.186-225.

[5] Packard Instrument Company, LSC-Cocktails Elemental Composition, LSC in Practice (1997).

[6] T. M. Undagoitia, F. von Feilitzsch, L. Oberauer, et al., Spectroscopy of electron-induced fluorescence in organic liquid scintillators, Eur. Phys. J. D57 (2010), pp.105-110.

[7] B. Ravat, H.Chapelotte, M. Grivet and A. Chambaudet, Model and simulation of quenching effects on the shape of a tritium $\beta^{-}$in a liquid scintillation counting, Comput. Phys. Commun. 127 (2000), pp.261-267.

[8] T. Aso, M. Hara, M. Shoji, et al., Systematic error of counting efficiency estimation due to spectral conformity assessment in Geant4 based liquid scintillation counter simulation, 2016 IEEE Nuclear Science Symposium and Medical Imaging Conference, N30-3 (2016).

[9] P. Skarstad, R. Ma and S. Lipsky, Organic Scintillators, Ed. D.L. Horrocks, Gordon \& Breach Science, New York, (1968), pp.1-422. 